Proceedings of the Online Conference "Applications of Physics in Mechanical and Material Engineering"

\title{
Physics of Acoustic Excitation on Boundary Layer Development
}

\author{
V. Sokolenko, W. Elsner*, \\ A. Dróżdż And R. GNATOWska \\ Częstochowa University of Technology, 42-201 Częstochowa, Poland \\ Doi: 10.12693/APhysPolA.139.613 \\ *e-mail: welsner@imc.pcz.pl
}

\begin{abstract}
In such a complex system as a modern turbofan engine, the laminar boundary layers developing on surfaces of turbine blades are exposed to strong perturbations caused by acoustic waves. A high concentration of acoustic energy inside such an engine, apart from other negative aspects such as fatigue loadings, can also have a major impact on the stability of the laminar boundary layer and can trigger an earlier laminar-turbulent transition. This work examines the effect of acoustic excitation at broadband frequency range on a boundary layer developing on a flat plate subjected to a weak adverse pressure gradient. The experiment was conducted with a low inlet turbulence intensity level ( $\mathrm{Tu}>1 \%)$ to provide a cleaner environment that magnifies the effects of the excitation frequency. It was shown that acoustic excitation can lead to a more rapid increase in flow instability followed by an earlier l-t transition. The paper also demonstrates that broadband acoustic excitation modifies the physics of the near-wall flows by amplifying the frequency which is the 2 nd harmonic of two-dimensional Tollmien-Schlichting $(\mathrm{T}-\mathrm{S})$ waves.
\end{abstract}

topics: boundary layer physics, acoustic waves, flow control, receptivity

\section{Introduction}

The performance of turbines and compressors in aero-engines depends on the characteristics of the boundary layer; especially the position of the laminar-turbulent zone plays an important role in the energy conversion process. It is well known that laminar-to-turbulent (l-t) transition is very sensitive to the surface roughness as well as to the level of external disturbances like free-stream turbulence or incident wakes. Typical far-field sound spectra of rotating machinery also contain tonal or broadband acoustic components [1]. Especially, the flow inside a modern turbofan engine is an example of the complex flow system, where laminar boundary layers developing on turbine blades' surfaces are exposed to strong perturbations caused by acoustic waves. A high concentration of acoustic energy inside such an engine, apart from other negative aspects such as fatigue loadings, can also have a major impact on the stability of the laminar boundary layer, particularly on triggering earlier $\mathrm{l}-\mathrm{t}$ transition [2]. This may lead to a significant lowering of engine operational performance characteristics as well as to an increased air pollution and noise.

The acoustic tones are commonly observed on airfoils operating in low Reynolds numbers, where a separation bubble occurs close to the trailing edge, where strong coherent perturbations are amplified in a separated shear layer, while broadband noise emissions are produced mainly for the attached flow at the trailing edge.

It can be expected that such an emission will affect the boundary layer on the profile and thus also the $1-t$ transition. Several studies indicate that such acoustic emissions commonly result from airfoil self-noise. In some cases, this effect can also propagate backwards and influence flow development via a feedback loop [3]. Although this phenomenon is very complex, the literature indicates that the available research is mostly devoted to the impact of acoustic excitation on the separated shear layer. Pröbsting and Yarusevych [4] found that such an impact, dictated either by suction or pressure side events, can alter separation bubble characteristics. On the other hand, Bernardini et al. [5] demonstrated that excitation at the most unstable frequency intensifies coherence of vortical structures shedding in the shear layer, thus increasing turbulent mixing which reduces the size of the separation.

Although some recent studies have addressed the possibility of flow control using acoustic excitation, the effect of sound on $\mathrm{l}-\mathrm{t}$ transition at the attached layer has not been thoroughly addressed. According to Zhou et al. [6], boundary layer transition may 
be regarded as the forced response of the laminar boundary layer to external disturbances, where the most important thing is the first stage, i.e., receptivity - the process which was first described by Morkovin [7]. This receptivity may occur, among others, due to sound wave interacting with the leading edge curvature, discontinuities in surface curvature or surface inhomogeneities. Jones et al. [8] showed that propagation of acoustic waves, especially in the low-turbulence environment, may occur at the selected frequency, which might be somewhat different from the one of the hydrodynamic instabilities. Therefore, it may be interesting to try to distinguish between these two impacts.

\section{Methodology}

Experimental investigations were performed at a facility equipped with an open-circuit wind tunnel and a specially designed test section located at the end of this tunnel equipped with a movable upper wall allowing to impose adverse pressure gradient (APG) conditions. A diagram of the test section, including the basic measuring apparatus is shown in Fig. 1. For the analyzed case, a boundary layer develops on the $1400 \mathrm{~mm}$ long flat plate, where a zero pressure gradient was maintained to a distance of $500 \mathrm{~mm}$ from the elliptical leading edge. Then, the inclination of the upper plate at an angle of $8 \mathrm{deg}$ forces a mild adverse pressure gradient.

To accomplish the flow control, a 15-inches 1-way speaker T115-800 (band: $100 \mathrm{~Hz}$ to $650 \mathrm{~Hz}$ ) is located at a certain distance from the tunnel outlet and pointed upward in the flow. The speaker was connected to Powersoft K 10 DSP amplifier driven by a signal generator controlled by the LabVIEW software and connected with a digital-analog National Instruments converter, reproducing a pink noise in a specified frequency range.

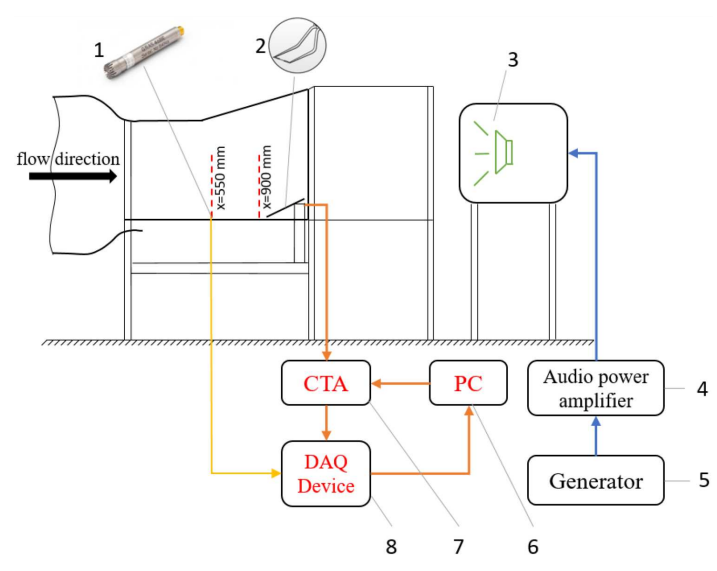

Fig. 1. Diagram of the test section and speaker location: 1 - microphone, 2 - single probe, 3 speaker, 4 - audio power amplifier, 5 - signal generator, 6 - PC, 7 - CTA, 8 - DAQ Device, $9-$ Lab VIEW.
Mean velocity in the core flow at the inlet plane to the test section was $U \approx 5 \mathrm{~m} / \mathrm{s}$. It corresponds to the Reynolds number $\operatorname{Re}_{x}=450000$ based on the length of the plate $x$. The freestream turbulence produced at the inlet $\mathrm{Tu}=0.6 \%$ and the integral length scale calculated from the autocorrelation of the velocity signal measured $80 \mathrm{~mm}$ upstream the flat plate leading edge equals $100 \mathrm{~mm}$. The velocity measurements were performed with the use of a hot-wire anemometry Dantec Dynamics Streamline Pro apparatus and a single hot-wire probe of a wire length of $l=1.25 \mathrm{~mm}$. A GRAS 46BE $1 / 4 "$ microphone connected to the National Instrument cDAQ-9174 system was used to perform sound field measurements in the test section. Based on the instrument's accuracy for a frequency range of $10-4000 \mathrm{~Hz}$, the uncertainty in the measured sound pressure levels (SPLs) is of $\pm 0.1 \mathrm{~dB}$ which is typical for such measuring devices [9].

In addition to the naturally developing flow, the flow exposed to the pink noise characterized by the sound pressure level SPL $=130 \mathrm{~dB}$ at frequency range $100-650 \mathrm{~Hz}$ was investigated. The pink noise is a random signal for which the spectrum density, i.e., narrow-band signal, varies as the inverse of frequency. This type of signal is used, among others, in the certification procedure of jet airplanes by the U.S. Federal Aviation Administration.

\section{Results and discussion}

The research described in this study was aimed at analyzing the response of the laminar boundary layer developing on the flat plate and to investigate the possibility of inducing an earlier l-t transition. Figure 2 depicts the distribution of one of the basic boundary layer parameters, that is, the shape factor $H$. The shape factor is defined as the ratio of integral parameters, displacement thickness to the momentum thickness and takes the value of 2.59 for a laminar (Blasius type) and 1.4 for a turbulent boundary layer. From $x=500 \mathrm{~mm}$, a clear increase in $H$ is seen, which is due to the influence of APG. For the unexcited case, $(N E)$ onset of the $1-\mathrm{t}$ transition is detected at the position $x=800 \mathrm{~mm}$, while for acoustic forcing $(E)$, it is shifted upstream by about $50 \mathrm{~mm}$. The analysis of the distributions in Fig. 3 indicates that the acoustic forcing has also some effect in the laminar boundary layer region, causing a slight decrease in the $H$ value already from the leading edge. However, the forcing is too small to destabilize the wall layer at an earlier position.

In order to investigate what is the physical mechanism of the acoustic wave impact, the characteristics of the boundary layer for two traverses were analyzed in more detail, i.e., for the so-called pseudolaminar region $(x=550 \mathrm{~mm})$ and for the transitional phase of the flow $(x=900 \mathrm{~mm})$. Figure 2 illustrates the mean streamwise velocity profiles and the streamwise Reynolds stress profiles for both 

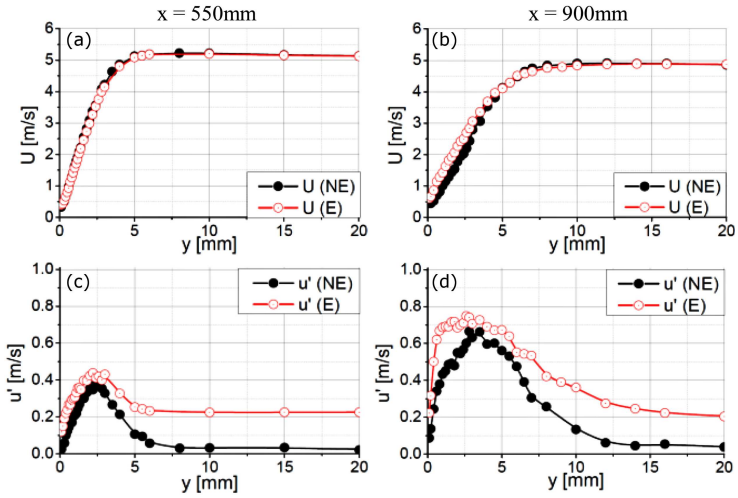

Fig. 2. (a, b) Mean velocity $U$ and (c, d) velocity fluctuation profiles $u^{\prime}$ without (filled circle) and with excitation (circle) measured for the laminar and transitional boundary layer.

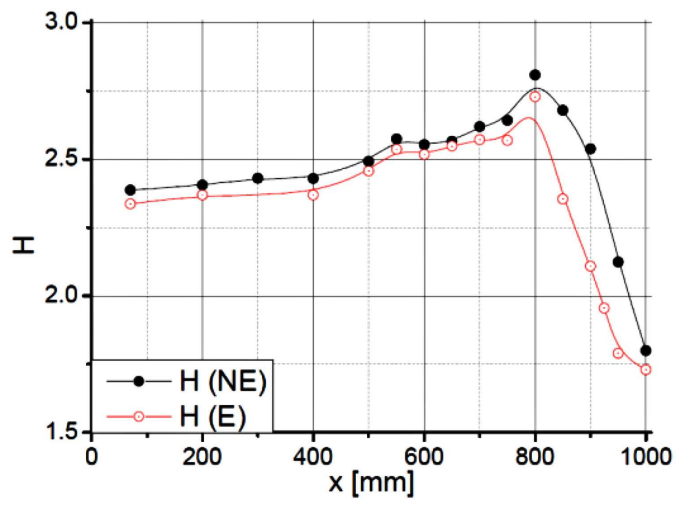

Fig. 3. Distribution of shape factor $H$ for noexcited $(N E-$ filled circle) and excited $(E-$ circle) boundary layer.

traverses. When looking at the mean velocity profiles, the effect of acoustic forcing is almost unnoticeable. However, it looks different in the case of the streamwise Reynolds stress.

As expected, for the naturally developing flow there is an almost twofold increase in boundary thickness $\delta$ from 6 to $13 \mathrm{~mm}$ and an increase of $u^{\prime}$ maximum by over $70 \%$. The most interesting fact, however, is that for the laminar boundary layer $(x=550 \mathrm{~mm})$, the acoustic excitation causes a strong increase (50-fold increase in energy) in the velocity fluctuation in the freestream from 0.032 to $0.224 \mathrm{~m} / \mathrm{s}$, while close to the wall the difference is almost negligible (only $30 \%$ increase in energy). This indicates a clear damping effect of the laminar boundary layer. A similar energy damping phenomenon occurs for the next traverse at $x=900 \mathrm{~mm}$. However, at this location it is observed apart from the region just near the wall $(y=0.1-3 \mathrm{~mm})$, where the level of fluctuation with forcing is nearly 2.5 times higher.

To further clarify the physical processes occurring in the boundary layer, a spectral analysis of the velocity signal recorded outside the boundary layer, i.e., $y=10 \mathrm{~mm}$, and in the shear layer near
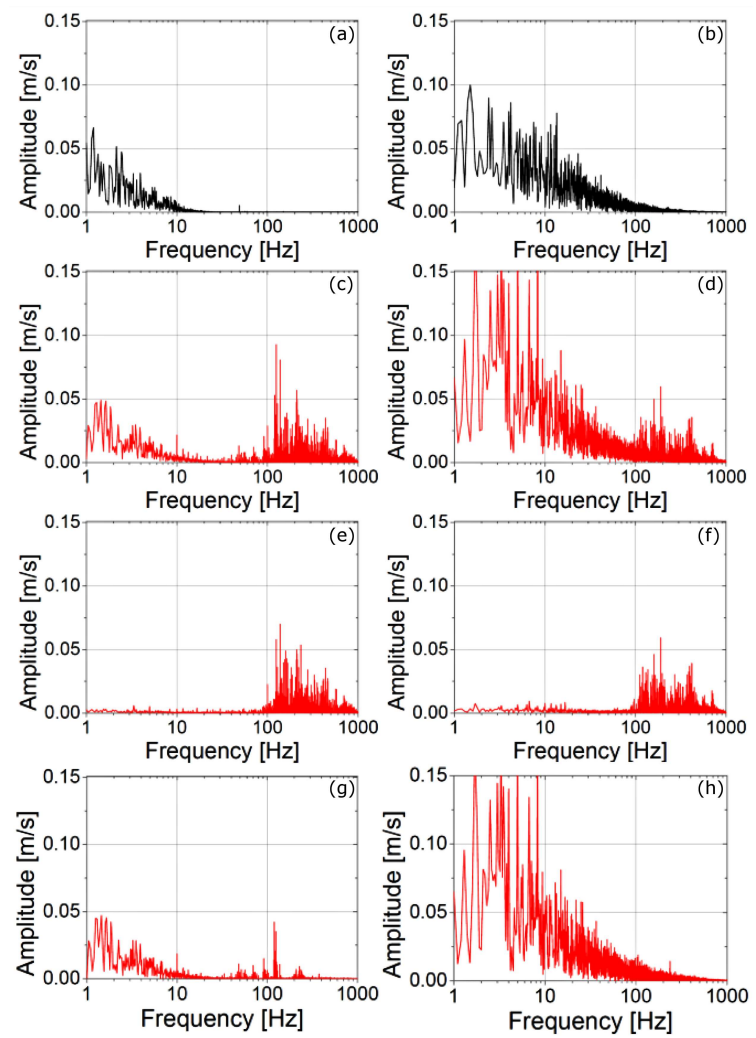

Fig. 4. Amplitude spectra for (a, b) no-exited $N E$ b.l., (c, d) exited $E$ b.l., (e, f) free field, (g, h) difference.

the wall, i.e., at the distance of $y=1 \mathrm{~mm}$, was performed (Fig. 4). In addition to the mentioned spectra, the difference spectra of the signal recorded inside and outside the boundary layer are shown in Fig. $4 \mathrm{~g}$, h. In the vicinity of the wall, for the unexcited case initially, the energy is concentrated in a very low frequency range while later, as a result of emerging instability and turbulence in the flow, the upper limit of the spectrum shifts towards higher frequency values. This is similar to the excited case, but here, in addition to the low-frequency fluctuations, the effect of acoustic forcing is seen in the $100-650 \mathrm{~Hz}$ band. This pattern is clearly visible in the freestream as it is depicted in the next set of graphs (Fig. 4e, f). The overall energy change in the analyzed signals can be estimated based on the RMS values summarized in Table I. The most important thing seems to be the last set of diagrams, i.e., the difference spectra, showing what is the share of the near wall energy due to external flow, including acoustic forcing, and what is the result of self-generated processes occurring in the wall layer. For $x=550 \mathrm{~mm}$, in addition to the lowfrequency fluctuations typical of a pseudo-laminar boundary layer, an amplification for a narrow frequency range around $120 \mathrm{~Hz}$ is evident.

According to Johnson and Pinarbasi [10], the laminar boundary layer is receptive to relatively narrow frequency bands. They identified two 
TABLE I level $(\mathrm{Tu}<1 \%)$ in order to provide a cleaner envi-

RMS of velocity signal recorded for $550 \mathrm{~mm}$ and $900 \mathrm{~mm}$. The notation "*" reads for $20 E$ for $x=900 \mathrm{~mm}$.

\begin{tabular}{l|c|c}
\hline \hline & $550[\mathrm{~mm}]$ & $900[\mathrm{~mm}]$ \\
\hline $1 N E$ & 0.210 & 0.433 \\
$1 E$ & 0.307 & 0.687 \\
$10 E^{*}$ & 0.224 & 0.204
\end{tabular}

frequency regimes: the first of them is responsible for the generation of Tollmien-Schlichting (T-S) waves and the second — for the generation of the elongated streaky structures, where the latter have spanwise wavelengths from approximately $\delta$ to $4 \delta$. The frequency of the T-S waves may be predicted by the linear stability theory, which involves a search for eigenvalues of the Orr-Sommerfeld equation. However, Walker [11], basing on Obremski's calculations, derived a correlation for the frequency of the most amplified $\mathrm{T}-\mathrm{S}$ waves in terms of the displacement thickness Reynolds $\delta^{*}$. This can be written as

$$
f=\frac{3.2}{2 \pi v} \frac{U_{\infty}^{2}}{\operatorname{Re}_{\delta *}^{3 / 2}},
$$

where $v$ is the kinematic viscosity. Based on the correlation (1), the frequency for $x=550 \mathrm{~mm}$ takes the value of $59.8 \mathrm{~Hz}$, which means that the excited frequency is the second harmonic of $\mathrm{T}-\mathrm{S}$ waves. These results also indicate that external forcing beyond this excited frequency has no noticeable effect on the laminar boundary layer. Further downstream, for $x=900 \mathrm{~mm}$ the amplification of fluctuation for the frequency $120 \mathrm{~Hz}$ is no longer observed. It is because the development of the two-dimensional $\mathrm{T}-\mathrm{S}$ waves is followed by the emergence of threedimensional structures. Thus, it can be hypothesized that at an earlier stage enhanced $\mathrm{T}-\mathrm{S}$ waves induced a faster destabilization of the boundary layer than the fluctuating energy is redistributed to all possible frequencies, which accelerates the $1-t$ transition. This also results in a faster increase in the momentum thickness and a consequent decrease in the shape factor $H$.

\section{Conclusions}

This work examines the effect of acoustic excitation at broadband frequency range on a boundary layer developing on a flat plate subjected to a moderate adverse pressure gradient. The experiment was conducted with a low inlet turbulence intensity ronment that magnifies the effects of the excitation frequency. It was shown that acoustic excitation can lead to a more rapid increase in flow instability followed by an earlier l-t transition. The paper also demonstrates that broadband acoustic excitation modifies the physics of the near-wall flows by amplifying the frequency which is the 2nd harmonic of two-dimensional Tollmien-Schlichting ( $\mathrm{T}-\mathrm{S})$ waves. The most effective frequency agrees with the inviscid stability theory and the higher amplification of waves takes place in the shear layer.

\section{Acknowledgments}

The investigation was supported by the National Science Centre under grant No. DEC2018/31/B/ST8/01717 and by the Ministry of Science and Higher Education of Poland from the funds dedicated to scientific research No. BS-PB-1-100$3011 / 2021 / \mathrm{P}$.

\section{References}

[1] W.D. Krusz, Z. Rarata, in: Proc. $23 r d$ AIAA/CEAS Aeroacoustics Conference, Denver (CO) $201 \%$.

[2] V.V Kozlov, in: Laminar-Turbulent Transition, 1985, p. 349.

[3] J.W. Kurelek, M. Kotsonis, S. Yarusevych, J. Fluid Mech. 853, 1 (2018).

[4] S. Pröbsting, S. Yarusevych, J. Fluid Mech. 780, 167 (2015).

[5] C. Bernardini, S. Benton, J.P. Bons in: Proc. Turbo Expo: Power for Land, Sea and Air, Copenhagen, 2012 p. 1559.

[6] M.D. Zhou, D.P. Liu, R.F. Blackwelder, Exp. Fluids 17, 1 (1994).

[7] M.V. Morkovin, in: Viscous Drag Reduction, Ed. C.S. Wells, Springer, Boston (MA) 1969, p. 1.

[8] L.E. Jones, R.D. Sandberg, N.D. Sandham, J. Fluid Mech. 648, 257, (2010).

[9] P. Niegodajew, K. Łukasiak, H. Radomiak, D. Musiał, M. Zajemska, A. Poskart, K. Gruszka, Combust. Flame 194, 245 (2018).

[10] M.W. Johnson, A. Pinarbasi, Flow, Turbul. Combust. 93, 1 (2014).

[11] G.J. Walker, AIAA J. 27, 595 (1989). 